\title{
Suicídio em jovens: fatores de risco e análise quantitativa espaço-temporal (Brasil, 1991-2001)
}

\section{Suicide in young people: risk factors and quantitaive space-time analysis}

\author{
Regina S. Rodrigues* \\ Ana Claudia F. M. Nogueira** \\ Jorge Antolini*** \\ Victor Berbara**** \\ Cátia Oliveira ${ }^{\star * * * *}$
}

\section{RESUMO}

O suicídio representa atualmente um sério problema de Saúde Coletiva, sendo a segunda causa de morte em jovens na Itália, na França e no Reino Unido e a terceira nos EUA. Este trabalho objetiva estudar a mortalidade por suicídio em adolescentes residentes nas cinco regiões do Brasil, comparativamente, no período de 1991 a 2001, revendo também os principais fatores de risco (FR) descritos na literatura. Metodologia: trata-se de um estudo epidemiológico, com enfoque sobre a Saúde Mental Juvenil. Foram utilizados os dados do Sistema de Informação de Mortalidade do Ministério da Saúde. Realizou-se a revisão da literatura por meio dos Sistemas Lilacs e Medline, além de livros temáticos. A discussão, de forma contextualizada, teve por base o Pensamento da Complexidade proposto por Edgar Morin. Resultados: foram registrados 69.811 óbitos por suicídio no período estudado, sendo 6.985 casos no grupo dos adolescentes. Os maiores Coeficientes de Mortalidade/100 mil habitantes ocorreram preponderantemente na região sul (1992 a 1994; 1996 a 1998; 2001), seguida pela região centro-oeste. Os principais FR descritos foram história de tentativas anteriores, ideação suicida, maus tratos, condições socioeconômicas precárias, doenças crônicas severas, impulsividade, depressão e esquizofrenia. O suicídio reflete características e valores relevantes de uma sociedade. Representa um sistema complexo e deve ser sempre analisado no contexto individual, social e coletivo. A atuação do profissional de Atenção Primária à Saúde (APS) é fundamental para a prevenção, por meio da identificação dos fatores e situações de risco, no contexto individual, familiar e comunitário.

\section{PALAVRAS-CHAVE:}

- Suicídio;

- Saúde do Adolescente;

- Epidemiologia;
KEY-WORDS:

- Suicide;

- Adolescent Health;

- Epidemiology;

\footnotetext{
* Médica, especialista pela Sociedade Brasileira de Pediatria e especialista em Epidemiologia aplicada à Saúde Mental, Universidade Federal do Rio de Janeiro, Rio de Janeiro, Brasil.

** Médica Clínica Geral, Secretaria Municipal de Saúde e Ministério da Saúde, Rio de Janeiro, Brasil.

*** Médico Clínico Geral, Hospital Universitário Gaffré Guinle, UNIRIO, Rio de Janeiro, Brasil.

**** Médico Epidemiologista, Secretaria Municipal de Saúde, Ministério da Saúde, Rio de Janeiro, Brasil.

${ }^{\star * * * *}$ Sanitarista, Mestre em Saúde Pública, Secretaria Municipal de Saúde e Secretaria de Estado de Saúde, Rio de Janeiro, Brasil.
} 


\section{ABSTRACT}

Suicide is today a huge public health problem (OMS, 2000). It is the second leading cause of death among young people in Italy, France and the United Kingdom and the third in the United States. The aim of this paper was to develop a comparative study of mortality from suicide in adolescents living in the five regions of Brazil during the period 1991-2001, and to review the risk factors $(R F)$ described in the literature. Methodology: this is an epidemiological study focusing on juvenile mental health using the data of the Mortality Information System of the Ministry of Health. The review of the literature was performed using the data- bases Lilacs and Medline as well as books dealing with the issue. The discussion is based on Edgar Morin's concept of "complexity" in a contextualized form. Results: 69.811 deaths from suicide were registered during the period under study, 6.985 from these in the group of adolescents. The highest mortality coefficients/100 thousand inhabitants occurred in the south of the country (1992 to 1994; 1996 to 1998; 2001), followed by the centralwest. The principal risk factors described in the literature were history of previous attempts, suicidal ideas, ill treatment, precarious socio-economic conditions, severe chronic disease, impulsivity, depression and schizophrenia. Suicide reflects characteristics and relevant values of a society. It represents a complex system that should always be analyzed in the context of the individual, the society and the community. The acting of the primary health care professional in the sense of identifying the risk factors in the individual, familiar and community context is fundamental in the prevention of suicide.

\section{Introdução}

A adolescência é um dos períodos de desenvolvimento caracteristicamente marcado por intensas transformações biológicas, sociais e psicológicas, sendo vulnerável a diversos agravos, inclusive relativos à saúde mental.

O suicídio representa atualmente um grave problema de Saúde Coletiva. Segundo a Organização Mundial de Saúde (OMS-2000) ${ }^{1}$, as estimativas realizadas indicam que, em 2020, as vitimas poderão ascender a 1,5 milhões. Estima-se ainda que um número de 10 a 20 vezes superior tentará o suicídio². Os jovens representam um grupo de elevada vulnerabilidade em diversos países: o suicídio representa a segunda causa de morte de jovens na Itália, na França e no Reino Unido e a terceira causa nos EUA, tendo dobrado a taxa de mortalidade entre 1960-2001 ${ }^{3}$

O suicídio é a principal causa de morte na Suíça, na faixa dos 14 aos 25 anos, correspondendo a um suicídio a cada três dias e 15 a 20 mil tentativas/ ano ${ }^{4}$.

No Brasil, os Coeficientes de Mortalidade por suicídio entre 1980 e 2000 foram considerados relativamente baixos quando comparados a outros países, ocupando $071^{\circ}$ lugar na classificação mundial, segundo a OMS ${ }^{1}$. Em jovens residentes em capitais brasileiras, estudo realizado por Souza et. al. ${ }^{5}$ apontou para uma certa elevação das taxas de suicídio entre 1979 e 1998, e, entre as causas externas, o suicídio ocupa a sexta posição.

Deve-se considerar, contudo, a baixa qualidade dos dados registrados no boletim de óbito como possível fator de subestimativas dos óbitos por suicídio no Brasil. 


\section{Objetivos}

Este trabalho objetiva estudar os Coeficientes de Mortalidade (CM) por suicídio em jovens no Brasil, no período de 1991 a 2001, nas diversas regiões do país, além de analisar os principais fatores e comportamentos de risco neste grupo etário, incluindo a ideação suicida e as tentativas, em uma abordagem reflexiva e transdisciplinar.

\section{Material e Método}

Trata-se de um estudo epidemiológico descritivo aplicado à Saúde Mental de Adolescentes. Para os cálculos de CM, foram utilizados os dados do Sistema de Informação de Mortalidade (SIM) referentes ao período de 1991 a 2001. As taxas foram calculadas por 100 mil habitantes. Foram analisadas as variáveis sexo e faixa etária em cada região do país, de forma comparativa, na construção desta série histórica.

Para a avaliação dos fatores e comportamentos de risco, realizou-se a revisão da literatura por meio dos Sistemas Lilacs e Medline, além de consulta de livros temáticos.

Considerando o suicídio resultante da interação de múltiplos fatores, optou-se, a nível reflexivo na discussão, pela Abordagem do Paradigma da Complexidade proposto por Edgar Morin.

\section{Resultados}

Segundo Friedrich, citado por Feijó, o "suicídio é cometido quando o indivíduo sente-se incapaz de dominar uma situação insuportável, tem a convicção de que não pode sair dela, desespera-se pela perda de controle sobre o ambiente; enfim, quando todas as maneiras de agir fracassaram"6.

Segundo a OMS ${ }^{1}$, a maioria dos indivíduos que cometeram suicídio apresentavam algum dos seguintes transtornos, em ordem decrescente de risco: depressão (todas as formas), transtornos de personalidade (anti-social e border-line com traços de impulsividade, agressividade e freqüentes alterações de humor, alcoolismo e especialmente para adolescentes, abuso de substância, esquizofrenia e problemas afetivos).

- Epidemiologia:

Foi analisada a série histórica de suicídios em adolescentes nas regiões brasileiras entre 1991 e 2001.

No período de 1991 a 2001 foram registrados 69.811 óbitos por suicídios no Brasil. Destes, 6.985 $(10,05 \%)$ ocorreram em adolescentes (entre 10 e 19 anos de idade).

Os Coeficientes de Mortalidade (CM)/100 mil habitantes para a população geral brasileira oscilaram entre 3,51, em 1991 e 1992 (menores índices), e 4,47, em 2001 (maior índice).

Em adolescentes, o menor $\mathrm{CM}$ foi registrado em $1992(1,49)$ e o maior, em $2001(2,26)$.

Quando são analisadas as cinco regiões brasileiras, para a população geral o maior CM ocorreu no sul em todos os anos da série, variando de 6,63, em 1993, a 8,86, em 1998. Em 2001, o CM desta região representou 1,89 vezes a taxa nacional $(8,47$ e 4,47, respectivamente).

A região centro-oeste ocupou o segundo lugar em CM em todo o período. Em 2001, último ano da série histórica, o CM foi de 5,78 (gráfico 1).

Para os adolescentes, o sul apresentou os maiores CM nos anos de 1992 a 1994, 1996 a 1998 e 2001, seguido pelo centro-oeste, o qual ocupou o primeiro lugar nos demais anos da série (gráfico 2).

Os menores CM, tanto para a população geral 
quanto para os adolescentes, ocorreram na região de 10 a 19 anos, os CM variaram de 0,65 (1992) a nordeste em todo o período estudado. Para a faixa 1,46(2001), representando um aumento de 2,24 vezes

\section{Coeficiente de Mortalidade por Suicídio/100.000 habitantes no Brasil e Regiões - População Geral 1991-2001}

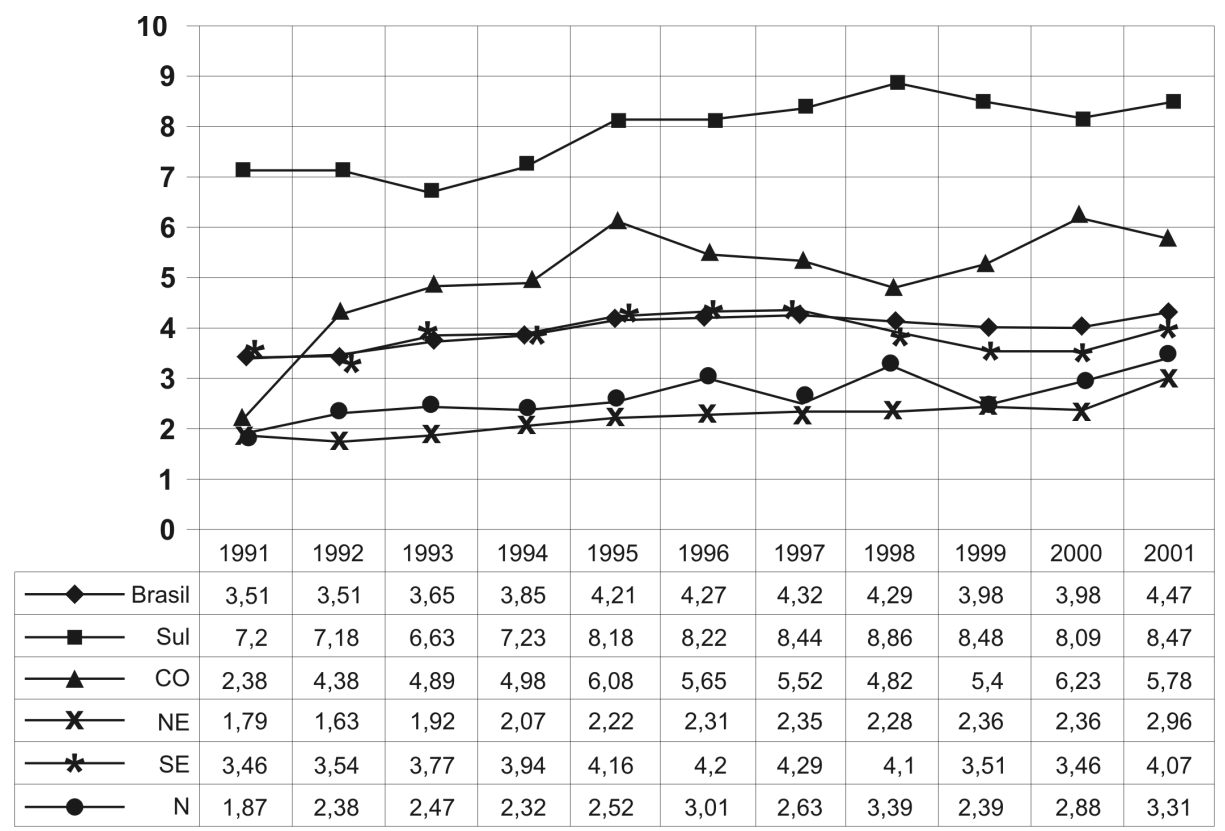

Fonte: Sistema de Informação de Mortalidade do Ministério da Saúde.

Coeficiente de Mortalidade por Suicídio por 100.000 habitantes no Brasil e Regiões - Adolescentes 1991-2001

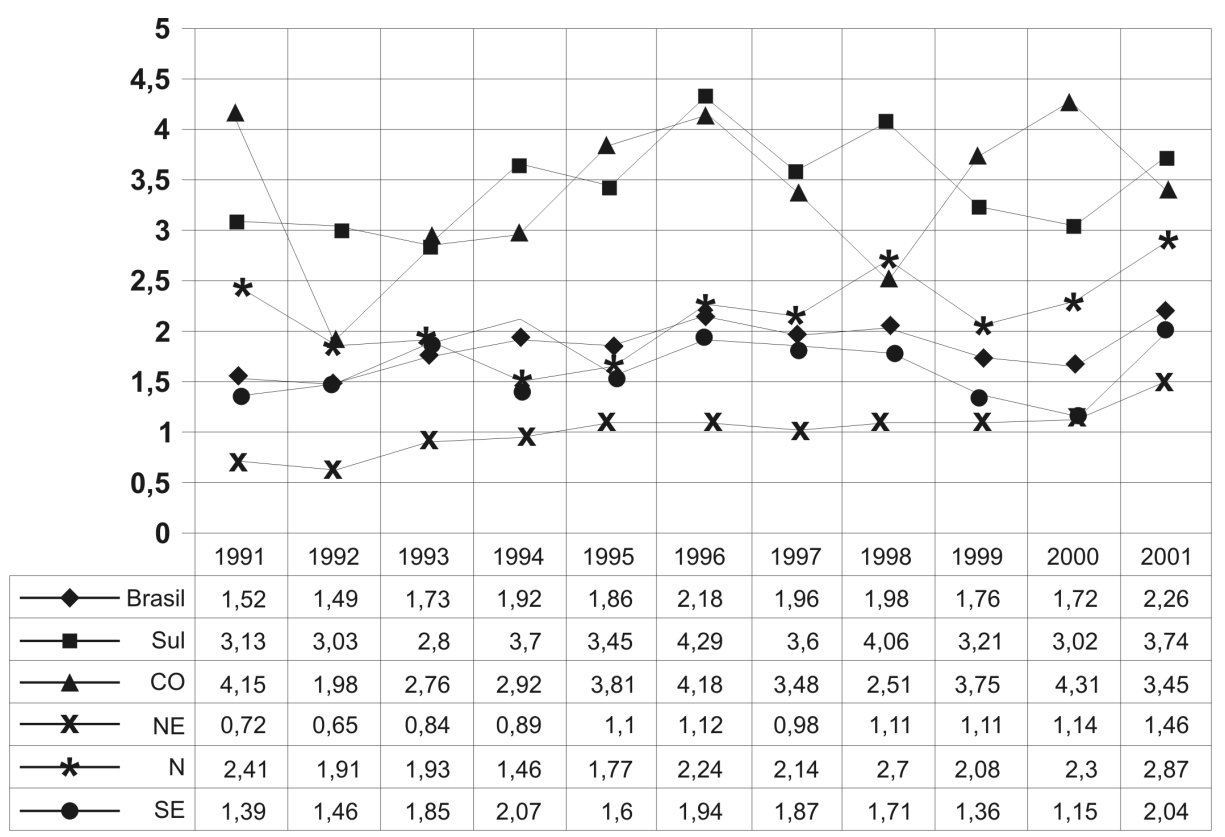

Fonte: Sistema de Informação de Mortalidade do Ministério da Saúde. 
nas taxas entre estes anos.

A região norte apresentou-se em terceiro lugar em relação aos CM mais baixos (exceto em 1994) para os adolescentes.

A região sudeste apresentou desenho da tendência próxima à curva nacional. Ocupou o terceiro lugar para os menores CM dentre as regiões para a população geral e o segundo para os adolescentes.

\section{Discussão}

O diagnóstico precoce do risco para suicídio é fundamental para a prevenção, sendo o profissional da APS o mais capacitado para perceber e atuar em tempo hábil: estudos realizados por Le Heuzey ${ }^{7}$ indicaram que $70 \%$ dos adolescentes haviam procurado um médico generalista ou da área de Saúde Escolar dias antes da concretização do ato.

A OMS sumariza os principais itens para avaliação e manejo de pacientes com risco para suicídio.

\section{Fatores de risco (FR) para o suicídio em jovens} Le Heuzey ${ }^{3}$ cita a seguinte classificação para avaliação de jovens com risco de suicídio:

- "Suicidé": é um indivíduo que alcançou a morte por

Risco de suicídio: identificação, manejo e plano de ação.

\begin{tabular}{|c|c|c|c|}
\hline $\begin{array}{l}\text { Risco de } \\
\text { Suicídio }\end{array}$ & Sintoma & Avaliação & Ação \\
\hline 0 & Nenhum & - & - \\
\hline 1 & $\begin{array}{l}\text { Com problemas } \\
\text { emocionais }\end{array}$ & $\begin{array}{l}\text { Perguntar sobre pensamentos } \\
\text { suicidas }\end{array}$ & Escutar com empatia \\
\hline 2 & $\begin{array}{l}\text { Idéias vagas de morte } \\
\text { suicidas }\end{array}$ & Perguntar sobre pensamentos & Escutar com empatia \\
\hline 3 & $\begin{array}{l}\text { Ideação suicida vaga } \\
\text { (plano e método) }\end{array}$ & Avaliar a intencionalidade & $\begin{array}{l}\text { Explorar as } \\
\text { possibilidades } \\
\text { Identificar apoio }\end{array}$ \\
\hline 4 & $\begin{array}{l}\text { Idéias suicidas } \\
\text { sem transtornos } \\
\text { psiquiátricos }\end{array}$ & $\begin{array}{l}\text { Avaliar a intencionalidade } \\
\text { (plano e método) }\end{array}$ & $\begin{array}{l}\text { Explorar as } \\
\text { possibilidades } \\
\text { Identificar suporte }\end{array}$ \\
\hline 5 & $\begin{array}{l}\text { Idéias suicidas e } \\
\text { transtornos } \\
\text { psiquiátricos ou fatores } \\
\text { estressores graves }\end{array}$ & $\begin{array}{l}\text { Avaliar a intencionalidade } \\
\text { (plano e método) }\end{array}$ & $\begin{array}{l}\text { Encaminhar para um } \\
\text { psiquiatra } \\
\text { Estabelecer um contrato }\end{array}$ \\
\hline 6 & $\begin{array}{l}\text { Idéias suicidas e } \\
\text { transtornos } \\
\text { psiquiátricos ou fatores } \\
\text { estressores graves ou } \\
\text { agitação e tentativas } \\
\text { prévias }\end{array}$ & $\begin{array}{l}\text { Ficar com o paciente } \\
\text { (para prevenir o acesso aos } \\
\text { meios letais) }\end{array}$ & Hospitalizar \\
\hline
\end{tabular}

Fonte: OMS, 2000. 
suicídio.

- "Suicidant": é o indivíduo que realizou pelo menos uma tentativa de suicídio.

- "Suicidaire": possui idéias de suicídio ou expressa desejos de suicidar-se.

\section{Tentativas}

Histórias de tentativas anteriores são um dos principais fatores de risco para o suicídio em qualquer grupo etário.

As tentativas são de 30 a 60 vezes mais freqüentes que o suicídio, sendo mais comuns em jovens que em idosos e ocorrendo principalmente no sexo feminino. A taxa de recidivas entre jovens é de 30 a $40 \%$, ocorrendo principalmente nos seis primeiros meses após a tentativa ${ }^{7}$.

Estudos apontaram que $56 \%$ dos suicidas morrem na primeira tentativa, especialmente os homens, com $62 \%$ de mortalidade comparados com $38 \%$ das mulheres. Dentre os sobreviventes, até $12 \%$ acabam se suicidando, representando um risco 38 vezes maior que a população em geral ${ }^{7}$.

\section{Ideação}

Estudos realizados por Rey Gex e analisados por Le Heuzey ${ }^{3}$ indicaram que entre 9.268 adolescentes, $26 \%$ apresentavam idéias suicidas. Estudos realizados por Miotto citado por Santos, Anelli e Sermous $^{8}$, em 2003, em adolescentes italianos de 15 a 19 anos, revelaram presença de ideação suicida em $30,8 \%$ das jovens e $25,3 \%$ dos rapazes.

Kandel, ao avaliar o risco para suicídio em 597 adolescentes, encontrou forte associação entre ideação suicida e uso de drogas. Em muitos casos houve relação entre o uso de drogas e sentimentos depressivos, principalmente em mulheres ${ }^{9}$.

\section{Maus-tratos na infância}

A violência durante a infância ou, às vezes, na própria adolescência representa considerável fator de risco (FR) para o suicídio. Estes jovens apresentam um risco três vezes superior de suicídio quando comparados a adolescentes sem história de violência física, sexual ou psíquica. Situações de abandono e frieza afetiva, principalmente por parte dos pais, também representam FR para o suicídio de jovens ${ }^{10}$.

\section{Condições socioeconômicas precárias}

Marx aponta as injustiças sociais e a opressão como causa de suicídio em mulheres jovens, citando diversos casos, representando um dos diversos sintomas das desigualdades sociais ${ }^{11}$. Estudos recentes corroboram esta análise ${ }^{7,12}$ : elevadas taxas de condições socioeconômicas precárias $(p<0,0001)$ estão associadas a doenças psiquiátricas $(p<0,0001)^{13}$.

\section{Doenças crônicas}

Doenças crônicas estigmatizantes, dolorosas e/ou incapacitantes também representam FR para o suicídio entre jovens. Dentre estas, destacam-se o diabetes mellitus, a insuficiência renal crônica, a asma, a obesidade e a mucoviscidose. Estudos realizados por Marcelli (1995) apontam para um processo de ódio contra o corpo-sede da doença e responsável pelos sofrimentos do adolescente ${ }^{7,11,14}$.

A AIDS representa importante fator de risco para o suicídio, o qual deve ser sempre investigado nestes pacientes ${ }^{8}$.

\section{Impulsividade}

Determinadas características comuns aos adolescentes, como a natural impulsividade que os leva a "atuar em um curto-circuito de pensamento 
transformado em ação", podem ser consideradas fatores suicidas ${ }^{10}$.

\section{Antecedentes familiares}

Estudos apontaram para $40 \%$ de estresse póstraumático e $31 \%$ de idéias suicidas em adolescentes após o suicídio realizado por familiar ${ }^{15}$.

\section{Suicídio de amigos e colegas}

Já foram relatadas "epidemias" de suicídio entre adolescentes após o suicídio de um amigo ou colega de escola, conhecido como "efeito Werther", em alusão ao clássico romance de Goethe, principalmente nos primeiros seis meses após o óbito. Nos dois anos seguintes, há um risco elevado de depressão, porém sem ideação suicida ${ }^{4,15}$.

\section{Drogas e álcool}

Um estudo realizado por Garfinkel et al. em 1982, em um hospital de emergências pediátricas, com 505 crianças e adolescentes internados por tentativa de suicídio, apontou uma elevada prevalência de uso abusivo de drogas e/ou álcool pelos familiares e/ou pelos próprios pacientes. A maior parte das tentativas ocorreu à noite, após a escola, na própria residência e devido à overdose de drogas ${ }^{16}$.

Alcoolismo é menos freqüente em adolescentes do que em adultos jovens, porém mais da metade das tentativas de suicídio envolveram ingestões prévias de álcool, que parece funcionar como fator de desinibição e impulsividade ${ }^{3}$.

A autópsia psicológica realizada por Shafü et al., em 20 crianças e adolescentes entre 12 e 19 anos que haviam cometido suicídio, revelou que $85 \%$ haviam manifestado ideação suicida, $40 \%$ já haviam cometido pelo menos uma tentativa, $70 \%$ abusavam de álcool e/ou drogas e 70\% tinham comportamento anti-social ${ }^{17}$.

Usuários de heroína apresentaram uma taxa 14 vezes maior para suicídio, segundo Darke et al. ${ }^{18}$.

Estudos realizados por Sher \& Zalsman apontaram para a elevada associação entre abuso de álcool e depressão em adolescentes suicidas ${ }^{17,19}$.

Fleischmann et al. observaram também que o abuso de substâncias estava presente em 40,8\% dos suicídios em jovens ${ }^{20}$.

O uso de drogas aumenta consideravelmente o risco de depressão, idéias, tentativas e suicídio consumado entre os jovens.

Segundo pesquisa coordenada pelo Centro para o Avanço da Ciência com 19 mil adolescentes de 132 escolas, os profissionais de saúde devem pensar seriamente no risco de suicídio em adolescentes que relatem episódios de uso de drogas ${ }^{21}$.

\section{Outros transtornos mentais - Patologias psiquiá- tricas}

Há uma elevada prevalência dos seguintes transtornos psiquiátricos em jovens suicidas, tais como a depressão, a ansiedade e o abuso de substâncias. A esquizofrenia e os transtornos alimentares também representam fatores de risco, segundo os estudos de Evans et al., em 200522.

\section{Depressão e suicídio em adolescentes}

Estudos epidemiológicos verificaram que 8,3\% dos adolescentes nos EUA apresentaram transtorno depressivo ${ }^{23}$, sendo que $4,9 \%$ podem apresentar a forma "major" da doença ${ }^{24}$.

Determinados transtornos do sono, como insônia e pesadelos, têm sido associados a um risco aumentado de suicídio. Contudo, parece que o link 
entre insônia e suicídio passa pela presença de transtornos depressivos ${ }^{25}$. O gesto suicida em jovens pode revelar uma esquizofrenia ou outro transtorno mental severo que até então encontrava-se silencioso $^{26}$

\section{Conclusão}

"O suicídio não é apenas um problema médico, mas de toda a sociedade"3.

Abordagens simplistas não possibilitam percepções profundas. A complexidade, enquanto teoria científica, inspira-se nos Sistemas Complexos. Paradigmas com base em visões unilaterais e certezas absolutas e simples não oferecem base segura à análise de situações tão complexas quanto o suicídio de jovens. Segundo Edgar Morin, "quando o simples já não basta, é preciso passar ao elo, à espiral, integrando a velha lógica ao jogo complexo". O todo é mais que a soma das partes: a abordagem do suicídio enquanto problema coletivo, dadas as cifras mundiais, requer a visão da parte (o indivíduo) na rede do todo (a família, a comunidade, a sociedade pós-moderna): as relações funcionam de forma complexa, ultrapassando a linearidade parte/todo, uno/diverso ${ }^{27}$.

Alguns fatores podem ser levantados como hipóteses explicativas para a diferença observada na mortalidade por suicídio entre os adolescentes residentes nas diversas regiões.

São relevantes as diferenças entre os Coeficientes de Mortalidade entre as regiões. Além dos fatores relativos à qualidade dos dados nos boletins de óbito, outros fatores podem, ao menos em parte, explicar as altas taxas na região sul: fatores de ordem afetivo-culturais e antropológicos, como explica Durkheim, citado por Silva, 2005. "Cada sociedade tem portanto, em cada momento de sua história, uma aptidão definida para o suicídio"28, além das baixas temperaturas (invernos mais rigorosos), fato amplamente estudado como "Depressão do Inverno" e/ou "Suicídio Sazonal"29,30,31.

As taxas relativamente baixas observadas nas regiões mais quentes, sob o ponto de vista climático (nordeste, norte e sudeste), poderiam corroborar a hipótese do efeito climático. Além disso, estas regiões são compostas na maioria dos estados, de clima afetivo mais caloroso, informal, cordial e predisposto ao lazer: fatores que podem ser considerados benéficos para um psiquismo resiliente ${ }^{12,32}$. Nos Estados Unidos da América, foram observadas baixas taxas de suicídio em adolescentes de origem hispânica quando comparados aos de origem anglo-saxônica, os quais apresentam taxas elevadas, apesar do melhor padrão aquisitivo e menor risco de discriminação social deste segundo grupo. Fatores protetores, como maior afetividade, ternura e apoio, são utilizados para justificar esta diferença, reforçando a importância do território familiar e comunitário para a saúde mental, principalmente em faixas etárias mais vulneráveis como a adolescência ${ }^{33}$. Estudos mais aprofundados, do tipo qualitativo, de base antropológica-cultural, psicossocial e psicológicas são necessários para compreender melhor estas diferenças entre os Coeficientes de Mortalidade entre as regiões.

Dessa forma, a abordagem inter e transdisciplinar, por meio do diálogo de respeito e abertura entre os diferentes olhares e distintos saberes (médico, psicológico, psicossocial, sociológico, antropológico etc.), torna-se fundamental para a compreensão e intervenção sobre o suicídio e tentativas a nível individual, familiar e comunitário. Como nos ensina Morin: "Hoje, a nossa necessidade histórica é encontrar um método capaz de detectar, e não ocultar, as 
ligações, as articulações, as solidariedades, as interdependências e as complexidades $34,35,36,37,38,39$.

A fragmentação do indivíduo dificulta a abordagem do mesmo como ser-de-relações: com sua família, com seu grupo social, com sua comunidade. A formação atual das Universidades das áreas Biomédicas não prepara os estudantes para serem profissionais capazes de abordar seus futuros pacientes de forma não-especializada. Fenômenos complexos, quando se transformam em epidêmicos ${ }^{1} a$ nível mental, exigem uma avaliação profunda, sem separar indivíduo e sociedade. E aí se encontra a questão do suicídio.

$\mathrm{Na}$ atual sociedade pós-moderna, na qual a produtividade e o progresso são valores essenciais, a morte prematura de jovens, que significa a opção voluntária pelo auto-extermínio, traduz questionamentos silenciosos e constitui um dos fenômenos mais intrigantes para os profissionais das áreas da saúde e sociais. O problema crucial é saber quando e de que forma indivíduos com potencial suicida transformarão suas fantasias ou ideações em atos concretos $^{27}$.

A intencionalidade revela o papel consciente do jovem para o auto-extermínio, a motivação revela as razões psíquicas para o desejo da morte, os precipitadores são os agentes, fatos ou circunstâncias, que, de maneira aguda ou crônica, considerando-se também o tipo de personalidade da vítima, "justificariam" o ato, e os métodos escolhidos indicam uma trajetória entre ideação, tentativas e morte opcional que deve ser percebida durante a avaliação do profissional de saúde responsável pelo paciente a fim de que medidas intervencionistas sejam instituídas em tempo hábil 17,40,41.

Este estudo revelou que os maiores $\mathrm{CM}$ no sul e os menores no nordeste podem indicar características socioculturais distintas, ocorrendo maior risco para o suicídio em adolescentes em culturas europeizadas. Contudo, deve-se atentar para a possibilidade de uma melhor qualidade dos dados nos estados do sul ${ }^{42}$.

Segundo Minayo et al., o diálogo entre os diferentes saberes, na busca da compreensão das interfaces entre os campos subjetivo e objetivo, possibilita uma abordagem mais profunda desse fenômeno ${ }^{43}$.

Os questionamentos sobre o auto-extermínio, principalmente em jovens, segundo Hillman, proporcionam a análise da intensa situação de sofrimento humano, tornando-se, assim, um "Paradigma de Vida"43.

Diversas especificidades situacionais, de cunho orgânico, familiar, antropológico-culturais e psicossociais podem associar-se como possiveis fatores desencadeantes da concretização do desejo de auto-extermínio, culminando no ato suicida. Este estudo não pode estabelecer qualquer relação por não dispor de dados relativos às questões subjetivas dos jovens que se mataram. "Para mensurar $o$ desespero humano e medir as potencialidades para o suicídio, individuais e grupais"14, abordagens qualitativas são necessárias.

Além disso, não se pode deixar de mencionar que óbitos por suicídio, devido ao tabu que cerca este evento, podem ser notificados no boletim de óbito como causa externa do tipo ignorado, induzindo à subnotificação do agravo, fato esse que pode ocorrer em qualquer região, mas principalmente naquelas de organização religiosa mais rigorosa.

Nosso estudo busca constituir um alerta para os profissionais de saúde que atendem crianças e 
adolescentes, na tentativa de diagnosticar precocemente situações de risco para o suicídio.

\section{Referências}

1. OMS. Prevenção do Suicídio: Um Manual para Profissionais de Saúde em Atenção Primária. [s.I.]: OMS; 2000.

2. Bertolote JM, Fleishmann A. A Global Perspective in the Epidemiology of Suicide. Suicidologia. 2002; 7(2): 6-7

3. Le Heuzey MF, Suicide de l'Adolescent. Ed. Le Quotidien du Medecin - Paris. 2001: 4-5; 5-8; Bah

4. Haemmerle $P$, Irminger $F$. Swissinfo - 13 de maio de 2005. Disponível em: http://www.swissinfo.org/por/ swissinfo.html. Acesso em: 12/06/06

5. Souza ER, Minayo MCS, Malaquias JV. Suicídio de jovens nas principais capitais do Brasil. Cad. Saúde Pública. [online]. 2002, vol. 18, no. 3 [citado 2006-0821], 673-683. Disponível em: <http://www.scielo.br/ scielo.php?script=sci_arttext\&pid=S0102.

6. Feijó M. Suicídio: Entre a Razão e a Loucura. São Paulo: Lemos Editorial; 1998. p.40.

7. Meleiro A, Teng CT, Wang YP. Suicídio - Estudos fundamentais Segmento. São Paulo: Fanna Editorial; 2004. p.125.

8. Santos O, Anelli M, Sermous G. Suicidi in Itália: Comportamenti e Credenze - Occhio Clínico n.9/ novembre 2004. Disponível em:

http://occhioclinico.it/occhio/2004/9articolo.php Acesso em: 03/08/06

9. Kandel DB, Ravels VH, Davies M. Suicidal Ideation. In: Adolescence: depression, substance use and other risk factors.

Disponível em: http://www.springerlink.com/content/ n77105867m1357w2/ Acesso em: 21/08/2006

10. Cassorla, RMS. Comportamentos Suicidas na
Infância e na Adolescência. In: Do Suicídio. São Paulo: Papirus; 1998. p. 61-87.

11. Marx K. Sobre o suicídio. São Paulo: Boitempo; 2006. p.13-30.

12. Chavagnat JJ. Prévention du Suicide. Motrouge (FR): John Libbey Eurotext; 2005. p.57-69.

13. Beautrais A, Joyce P, Mulder R. Risk Factors for serious Suicide Attempts among youths aged 13 through 24 years. Journal of the American Academy of Child and Adolescent Psychiatry. sept.1996; 35(9):1174-1182.

14. Kurella M, Kimmel PL, Young BS, Chertow GM. Suicide in the United States End Stage Renal Disease Program. J Am Soc Nephrology. 2005; 16:774-781. 15. Brent D, Bridge J, Connolly J. Suicidal Behavior runs in families: A Controlled Family Study of Adolescent Suicide Victims. Arch Gen Psychiatry. 1996; 53: 1145-1152.

16. Garfinkel BD, Froese A, Hood J. Suicide Attempts in Children and Adolescents. Am J Psychiatry. 1982;139:1257-1261.

17.Shafü $M$, Carrigan S, Whittinghill JR, Derrick A. Psycological Autopsy of Completed Suicide in Children and Adolescents. Am J Psychiatry.1985; 142:10611064. Disponível em: http://ajp.psychiatryonline.org/cgi/ content/abstract/142/9/1061 Acesso em: 11/06/2006 18. Darke S, Ross J. Suicide Among Heroin Users: Rates, Risk Factors and Methods. "Addiction". Disponível em: http://www.blackwell-synergy.com/ links Acesso em: 12/07/2006

19. Sher L, Zalsman G. Alcohol and Adolescent Suicide. Int J Adolesc Med Health; Jul-Sep. 2005; 17(3):197-203.

20. Fleischmann A, Bertolote JM, Belfer M, Beautrais A. Complete Suicide and Psychiatric Diagnoses in Young People: a Critical Examination of the Evidence. Am J 
Orthopsychiatry. Oct. 2005; 75(4): 676-83.

21. FAPESP Notícias - Fundação de Amparo à Pesquisa do Estado de São Paulo. Disponível em: http:// www.fapesp.br Acesso em: 14/09/2004

22. Evans DI, Foa EB, Gur R E, Hendin H, O’Brian CP, Seligman MEP, Walsh BT. Treating and Preventing Adolescent Mental Health Disorders: What we know and what we don't know. Am J Psychiatry. Apr. 163:4; 206:753-754.

23. Weissman MM, Wolk S, Goldtein RB, Moreau D, Adams P, Greenwald S, Klier CM, Ryan ND, Dahl RE, Wickramaratne P. Depressed Adolescent and Grown up. J Am Med Assoc. May. 1999; 281(18): 1707-1713.

24. National Institute of Mental Health. USA: NIMH; Sept 2000.

25. Liu X, Buysse D. Current Opinion in Psychiatry. may. 2006; 19(3): 288-293.

26.Cunha JA. Psicodiagnóstico. Porto Alegre (RS): Artmed; 2000. p.196-201.

27. Morin E. Le Paradigme Perdu: La Nature Humaine. Paris : Du Sevil; 1973. p.16-37.

28. Silva AO. Suicídio, Literatura e Sociologia. Revista Espaço Acadêmico. Disponível em: http// www.espacoacademico.com.br/044/44eozai.htm Acesso em: 06/02/07.

29. Breuer HW, Breuer J, Fischbach-Breuer BR. Social, toxicological and meteorological data on suicide attempts. Eur Arch Psychiatry Neurol Sci. 1986; 235(6): 367-70.

30. Nosad S. Dépression Saisonnièr :Blues de l'hiver : les enfants et les adolescents peuvent aussi souffrir de la Déprime Hivernale. Disponível em: http// www.alpha_lite.com/depression_saisonniere_enfant_ adolescents.pdf - Acesso em: 06/02/07.

31.Seminário Nacional dePrevenção ao Suicídio PUC/RS - Porto Alegre - 17 e 18/08/06
32. Mimouni M, Dellad JS, Kebdani K. Le Suicide Chez les Jeunes de 15 à 25 ans dans la Wilaya d'OranGuide des Recherches et des Chercheurs 2003-2004 - Centre de Recherche en Anthropologie Sociale et Culturelle. Disponível em:

http//www.crasc.org/contacts/projets_etablis/ projet20.php Acesso em: 06/02/07.

33. Castro JZ. O Suicídio entre Adolescentes Americanos. Revista Espaço Acadêmico Disponível em: http//www.espacoacademico.com.br/044/ 44eozai.htm Acesso em: 06/02/07.

34. Morin E. O Pensar Complexo. Rio de Janeiro: Garamond ; 1999. p. 21-34.

35. Morin E. Ciência com Consciência. São Paulo: Bertrand Brasil; 1996. p.119-123; 135-156; 252-275. 36. Morin E. Saberes Globais e Saberes Locais: o olhar transdisciplinar. Rio de Janeiro: Garamond; 2001. p.35-68.

37. Pena V. O Despertar Ecológico: Edgar Morin e a Ecologia Complexa. Rio de Janeiro: Garamond; 2003. p.19-44.

38. Costa C. Sem medo do Complexo. Idéias Livros. Jornal do Brasil. 12 de jun. 1999.

39. Teixeira E. As Três Metodologias: acadêmica, da ciência e da pesquisa. Petrópolis (RJ): Vozes; 2005. p. $94-114$.

40. Fairbairn GJ. Reflexões em Torno do Suicídio: a lingüagem e a ética do dano pessoal. São Paulo: Paulus; 1999.

41. Rodrigues RS, Antolini JL, Berbara VA, Oliveira C. Suicídio de Adolescentes no Brasil: diferenças regionais. 2006. Anais do último Congresso de MGC / SF. Jun. 2006.

42. Minayo MCS, Cavalcante FG, Souza ER. Proposta Metodológica para a Abordagem do Suicídio como Fenômeno Complexo. Cad Saúde Pública. ago. 2006; 
22(8): 1587-1596.

43. Hillman J. Suicídio e Alma. Petrópolis (RJ): Vozes;

1998. p.49-60.

Endereço para correspondência:

Ana Claudia F. M. Nogueira

Rua Conde de Bonfim, n 370/815

Rio de Janeiro - RJ

CEP: 20520-054

\section{Endereço Eletrônico:}

anaclaudiafmnogueira@ig.com.br 